\title{
The Tully-Fisher relation: evolution with redshift and environment
}

\author{
Alfonso Aragón-Salamanca \\ School of Physics and Astronomy, University of Nottingham, University Park, Nottingham, \\ NG7 2RD, UK \\ email: alfonso.aragon@nottingham.ac.uk
}

\begin{abstract}
The Tully-Fisher Relation (TFR) links two fundamental properties of disk galaxies: their luminosity and their rotation velocity (mass). The pioneering work of Vogt et al. in the 1990's showed that it is possible to study the TFR for spiral galaxies at considerable look-backtimes, and use it as a powerful probe of their evolution. In recent years, several groups have studied the TFR for galaxies in different environments reaching redshifts beyond one. In this brief review I summarise the main results of some of these studies and their consequences for our understanding of the formation and evolution of disk galaxies. Particular emphasis is placed on the possible environment-driven differences in the behaviour of the TFR for field and cluster galaxies.
\end{abstract}

Keywords. galaxies: formation; galaxies: evolution; galaxies: kinematics and dynamics

\section{Introduction}

The Tully-Fisher relation (TFR; Tully \& Fisher 1977) is a strong correlation between the luminosity and the maximum rotation velocity of spiral galaxies. Although its physical origin is not completely understood in detail, the TFR has proved to be a very useful tool to study the evolution of the fundamental properties of spiral galaxies. Vogt et al. (1996) showed that using large telescopes with efficient spectrographs it is possible to study the TFR reaching $z \sim 1$. Following that work, several groups have carried out similar studies for sizeable samples of disk galaxies over a wide range in redshift and environment.

\section{Evolution of the Tully-Fisher Relation of field galaxies}

The optical TFR of field galaxies has been studied using slit spectroscopy obtained at the VLT, Subaru and Keck telescopes by several groups including Vogt et al. (1996), Ziegler et al. (2002), Böhm et al. (2004), Bamford et al. (2006), Nakamura et al. (2006) and Weiner et al. (2006). These studies find that at a fixed rotation velocity, spiral galaxies appear to be $\sim 0.5-1.5 \mathrm{mag}$ brighter at $z \sim 1$ than at $z \sim 0$ in rest-frame $B$. If interpreted as pure luminosity evolution, this brightening implies a factor of $\sim 3$ increase is star-formation rate between $z \sim 0$ and $z \sim 1$ (Bamford et al. 2006; Nakamura et al. 2006). However, the sample selection and the exact details of how the comparison is made between samples at different redshifts have very severe effects on the measured evolution (cf. Vogt 2006, this symposium). Böhm et al. (2004) found a correlation between the optical TFR residuals and the rotation velocity $V_{\text {rot }}$ and argued that the observed luminosity evolution is mass dependent, in the sense that low-mass galaxies seem to exhibit a much stronger rate of evolution than massive ones (but see Weiner et al. 2006 for an opposite view). However, Bamford et al. (2006) demonstrated that such a correlation may exist purely due to an intrinsic coupling between the $V_{\text {rot }}$ scatter and the TFR 
residuals, acting in combination with the selection effects inherent to magnitude-limited samples. Thus, decoupling real evolution from observational artifacts if far from trivial.

Studies at longer wavelengths (e.g., Conselice et al. 2005) find very little or no evolution in the near-infrared (or stellar mass) TFR of field spirals up to $z \sim 1$, suggesting that baryonic mass is accreted by disks along with dark matter at $z<1$.

\section{The effect of the cluster environment on the Tully-Fisher Relation}

Kannappan et al. (2002) found that the deviations from the TFR of nearby galaxies are strongly correlated with their colours and $\mathrm{H} \alpha$ equivalent widths, and thus with their star formation properties and history. Correlations with the degree of photometric and kinematic asymmetry were also found, presumably linked with the dynamical interactions and history of the galaxies. Since these effects are environmentally-dependent, it is clear that the TFR and its evolution can be strongly influenced by the environment.

Vogt et al. (2004a,b,c) carried out a detailed study of the effect of the cluster environment on the rotation curves and TFR of low redshift galaxies. One of their results is that cluster and field star-forming spirals follow essentially the same TFR, while nonstarforming galaxies seem to be too faint for their rotation velocity. These spirals could be on their way to becoming S0s (see $\S 4$ ).

Comparisons of the TFRs of cluster and field spirals at redshifts reaching $z \sim 1$ have been published by Milvang-Jensen et al. (2003), Ziegler et al. (2003), Bamford et al. (2005), Nakamura et al. (2006) and Metevier et al. (2006). Although most of these studies do not find significant differences between the TFRs of field and cluster spirals, MilvangJensen et al. and Bamford et al. reported some evidence suggesting that cluster starforming spirals are brighter, at a fixed $V_{\text {rot }}$, than their field counterparts at the same redshift. Their interpretation is that field spirals falling into rich clusters undergo a (short?) period of enhanced star formation before having their star formation quenched by the cluster environment and transforming, perhaps, into S0s. Nakamura et al. (2006) showed that even if such processes are taking place, their effect on the TFR of starforming cluster galaxies could be quite difficult to detect. They also demonstrated that the sample selection and the details of how the analysis is actually carried out could have a very strong effect on the conclusions of cluster-field comparisons.

\section{The Tully-Fisher Relation of lenticular (S0) galaxies}

In recent years several groups have extended the study of the TFR to S0 galaxies (see Bedregal et al. 2006a,b and references therein). Despite the considerable technical challenges involved in determining the true $V_{\text {rot }}$ from absorption line spectra of galaxies with significant non-rotational support (see Mathieu et al. 2002), it seems clear now that S0s are, at a given rotation velocity, fainter than spiral galaxies in the optical and near-infrared. The measured offset between the SO and spiral TFRs seems now to be consistent with the hypothesis that S0s where once spirals that ceased forming stars. Indeed, the amount of fading implied by the offset from the spiral TFR of individual S0 galaxies seems to correlate with how long ago their star formation ceased (see figure 1 and Bedregal et al. 2006b). Interestingly, a similar conclusion is reached from the properties of the globular cluster systems of S0s and spirals (Aragón-Salamanca et al. 2006).

\section{Conclusions and future prospects}

Much has been learned so far on how the TFR of disk galaxies changes with environment and redshift, but significant uncertainties still remain. Progress is being made from larger galaxy samples like the ones now available in the field (e.g., Weiner et al. 

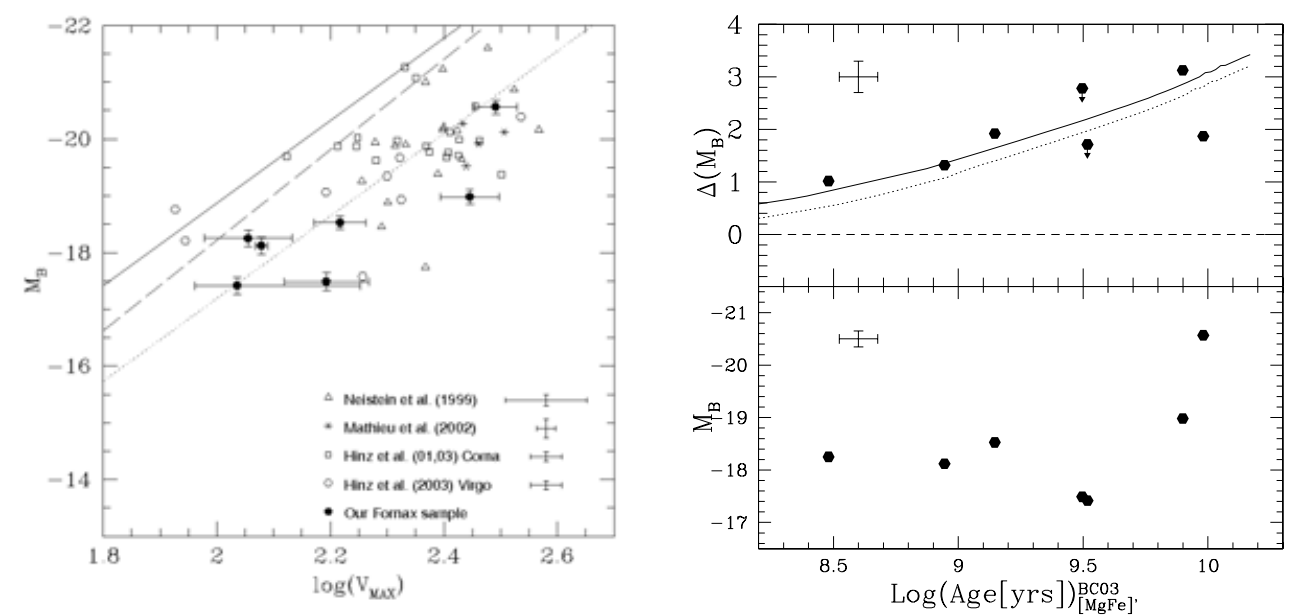

Figure 1. (Left) B-band Tully-Fisher relation of S0 galaxies (datapoints). Solid and dashed lines represent two alternative determinations of the TFR of spiral galaxies, while the dotted line is a fit to the $\mathrm{S} 0$ data. (Right) The offset from the spiral TFR $\left(\Delta M_{\mathrm{B}}\right)$ and absolute $B$ magnitudes $\left(M_{\mathrm{B}}\right)$ versus the Lick-indices determined ages of a sample of $\mathrm{S} 0$ galaxies in the Fornax cluster. The solid and dotted lines show population synthesis model predictions for fading spiral galaxies. See Bedregal et al. (2006a,b) for details.

2006) and in clusters (e.g., the ESO Distant Cluster Survey, White et al. 2004). Based on these samples, new TFR studies comparing a range of field and cluster environments will soon become available (Milvang-Jensen et al., Bamford et al., in preparation). Integral Field Spectroscopy (IFS) is adding another dimension to our understanding of the TFR and the physical causes of its scatter and evolution (cf. Flores et al. 2006). IFS coupled with the magnification provided by gravitational lensing is helping us reaching larger redshifts and higher spatial resolutions, although the samples are still small (Swinbank et al. 2006). Extending the redshift baseline requires the efficient near-infrared spectrographs (Smith et al. 2004, van Starkenburg et al. 2006) now becoming available at large telescopes.

\section{Acknowledgements}

I thank S. Bamford, A.G. Bedregal, M. Merrifield, B. Milvang-Jensen, O. Nakamura, N. Arimoto, C. Ikuta and L. Simard for allowing me to discuss here results obtained with their help.

\section{References}

Aragón-Salamanca, A., Bedregal, A. G., \& Merrifield, M. R., 2006, A\& A, 458. 101

Bamford, S.P., Milvang-Jensen, B., Aragón-Salamanca, A., \& Simard, L. 2005, MNRAS, 361,109

Bamford, S. P., Aragón-Salamanca, A., \& Milvang-Jensen, B. 2006, MNRAS, 366, 308

Bedregal, A. G., Aragón-Salamanca, A., Merrifield, M. R., \& Milvang-Jensen, B. 2006a, MNRAS, 371,1912

Bedregal, A. G., Aragón-Salamanca, A., \& Merrifield, M. R., 2006b, MNRAS, in press, astro$\mathrm{ph} / 0609076$

Böhm, A., et al. 2004, A\&\&A, 420, 97

Conselice, C. J., Bundy, K., Ellis, R. S., Brichmann, J., Vogt, N. P., \& Phillips, A. C. 2005, ApJ, 628, 160

Flores, H., Hammer, F., Puech, M., Amram, P., \& Balkowski, C. 2006, A\&AA, 455, 107

Kannappan, S. J., Fabricant, D. G., \& Franx, M. 2002, AJ, 123, 2358

Mathieu, A., Merrifield, M. R., \& Kuijken, K. 2002, MNRAS, 330, 251 
Metevier, A. J., Koo, D. C., Simard, L., \& Phillips, A. C. 2006, ApJ, 643, 764

Milvang-Jensen, B., Aragón-Salamanca, A., Hau, G. K. T., Jørgensen, I., \& Hjorth, J. 2003, $M N R A S, 339, \mathrm{~L} 1$

Nakamura, O., Aragón-Salamanca, A., Milvang-Jensen, B., Arimoto, N., Ikuta, C., \& Bamford, S. P. 2006, MNRAS, 366, 144

Smith, J. K., et al. 2004, MNRAS, 354, L19

Swinbank, A. M., Bower, R. G., Smith, G. P., Smail, I., Kneib, J.-P., Ellis, R. S., Stark, D. P., \& Bunker, A. J. 2006, MNRAS, 368, 1631

Tully, R. B., \& Fisher, J. R. 1977, A\&A A, 54, 661

van Starkenburg, L., van der Werf, P. P., Yan, L., \& Moorwood, A. F. M. 2006, A\& A, 450, 25

Vogt, N. P., Forbes, D. A., Phillips, A. C., Gronwall, C., Faber, S. M., Illingworth, G. D., \& Koo, D. C. 1996, ApJ, 465, L15

Vogt, N. P., Haynes, M. P., Herter, T., \& Giovanelli, R. 2004a, AJ, 127, 3273

Vogt, N. P., Haynes, M. P., Giovanelli, R., \& Herter, T. 2004b, AJ, 127, 3300

Vogt, N. P., Haynes, M. P., Giovanelli, R., \& Herter, T. 2004c, AJ, 127, 3325

Weiner, B. J., et al. 2006, ApJ, in press, astro-ph/0609091

White, S. D. M., et al. 2005, A\&A, 444, 365

Ziegler, B. L., et al. 2002, ApJ, 564, L69

Ziegler, B. L., Böhm, A., Jäger, K., Heidt, J., Möllenhoff, C. 2003, ApJ, 598, L87

\section{Discussion}

VogT: Would you care to speculate on the formation mechanisms for the high luminosity end of the S0 distribution?

ARAgón-SAlamancA: They are probably not formed by disk galaxy infall at $z \sim 0.5$. The more massive ones are probably in place quite early, but we do not really have much observational evidence on how they formed.

BUREAU: What are the prospects/future offered by integral field spectroscopy?

Aragón-Salamanca: As the work of Flores et al. and Swinbank et al. shows, integral field spectroscopy can provide not only an "integrated" rotation curve, as long-slit spectroscopy does, but also detailed kinematical information. This additional information allows us to find out, for instance, whether galaxies which deviate from the TFR do so because of straightforward luminosity evolution, of because of peculiar/disturbed kinematics. Thus we can not only measure evolution/deviations from the TFR, but also get a handle on the physical causes.

SADLER: Just a comment - the original TFR in the local universe was built on radio observations of the $21 \mathrm{~cm}$ HI line. Radio HI studies at $z>0.1$ are only just becoming possible, but in the next 5 years it should be possible to measure HI rotation curves out to $z \simeq 0.5$ with the technology prototypes currently being built for the Square Kilometre Array. As the collecting area increases it should eventually be possible to extend these studies for $z \simeq 1$ and beyond.

Aragón-SAlamancA: I agree. Although this review concentrates mainly on optical and near-IR studies (the ones that today are able to reach high enough redshifts for evolutionary studies), I believe radio studies have the potential to contribute greatly in this field. Indeed, at low redshifts HI observations are already telling us a lot about how the environment affects galaxy properties. 